\title{
Numerical Modelling of Wave Fields and Currents in Coastal Area
}

\author{
Francesco Gallerano $\mathbb{D}$ \\ Department of Civil, Constructional and Environmental Engineering, "Sapienza" University of Rome, \\ 00184 Roma, Italy; francesco.gallerano@uniroma1.it; Tel.: +39-064-458-5062
}

Received: 25 May 2020; Accepted: 1 June 2020; Published: 2 June 2020

\begin{abstract}
The design and management of coastal engineering, like harbors and coastal defense structures, requires the simulation of hydrodynamic phenomena. This special issue collects five original papers that address state of the art numerical simulations of wave fields and wave-induced velocity fields in coastal areas. The first paper proposes a turbulence model for wave breaking simulation, which is expressed in terms of turbulent kinetic energy and dissipation rate of turbulent kinetic energy $(k-\varepsilon)$; the proposed turbulence model is a modification of the standard $k-\varepsilon$ turbulence models. The second paper investigates modalities by which wind interacts with wave motion, modifying the wave propagation dynamic. The third paper proposes a study on waves overtopping over coastal barriers. The fourth paper details the numerical simulation of a tsunami wave that propagates over an artificial reservoir, caused by a landslide that creates a solid mass to detach from the slopes and to slide into the reservoir. The fifth paper examines an application case concerning Cetraro harbor (Italy), which is carried out using three-dimensional numerical simulations of wave motion.
\end{abstract}

Keywords: three-dimensional free surface flow simulation; waves and currents; turbulence model; landslide tsunami; wind and wave breaking; wave overtopping.

\section{Introduction}

The simulation of wave fields and wave-induced velocity fields is necessary to evaluate the actions exerted by the sea on coastal structures, as well as to forecast the effects produced by these structures on the morphological evolution of shorelines. Besides exerting a mechanical pull on coastal structure, wave motion and wave-induced velocity fields are responsible for coastal sediment transport phenomena. This special issue focuses on the numerical simulation of wave fields and wave-induced velocity fields in coastal areas. The topics of this special issue range from new numerical schemes for wave propagation simulation; evolutions from deep water to shoreline; numerical investigations regarding specific problems like wave-breaking, turbulence models, wind effects on wave propagation over a sloping bed, wave overtopping, and dynamics related to wet-dry front propagation. In the models presented in this special issue [1-5], the motion equations are three-dimensionally (3D) solved.

In recent literature [6-8], numerical simulations of waves and coastal currents have been carried out using models based on depth-averaged two-dimensional motion equations. In the simplest models, governing equations are represented by nonlinear shallow water equations (NSWE). In numerical shock-capturing schemes, energy dissipation caused by wave breaking is represented by energy dissipation associated with shallow water shock. NSWE-type models use shock-capturing schemes and are able to simulate wave breaking. Unfortunately, these models' frequency dispersion terms are neglected and thus cannot be used to represent phenomena limited to surf and swash zones. A more recent class of depth-averaged models solves the Boussinesq equations (BEs). In these equations, higher order terms are retained via a power expansion of hydrodynamic variables along a vertical 
direction. Models based on BEs incorporate frequency dispersion and are thus able to represent wave propagation in deep and intermediate water. Chen et al. [9] improved the abovementioned models by introducing an additional term to the momentum equation, which ensured the property of vertical vorticity conservation and improved surface wave and longshore current simulation. The presence of the BEs dispersive terms balances amplitude dispersion phenomenon and makes wave fronts more stable. For this reason, BEs are not able to automatically and intrinsically represent shallow water wave breaking. Consequently, in models based on BEs, the dispersive terms are switched off at the beginning of the breaking zone, which reduce BEs to NSWE. These models are able to simulate wave breaking thanks to the use of shock-capturing schemes. Still, they have the drawback of defining an "a priori" criterion, wherein it is decided when and where the wave breaking begins. The more recent works that feature BEs are Ref. [10-14]. In particular, in [10] broken waves characteristics in front of a vertical seawall are modelled and studied using a shock-capturing Boussinesq wave model. [11] presents a shock-capturing numerical model based on extended BEs suitable for rapidly varying topography with respect to wave transformation, breaking, and runup. In [12], a two-dimensional wave model coupled with ice dynamics is developed to evaluate ice effects on shallow water propagation on a beach and in a channel. The study proposed by [13] utilizes a shock-capturing Boussinesq model to investigate the maximum momentum flux in the solitary wave runup zone over back-reef slopes. [14] simulates the tsunami propagation and flooding in Sicilian coastal areas.

Models that solve three-dimensional motion equations for the simulation of free-surface flows-in which the dynamic pressure component is taken into account-are able to simulate the wave motion in deep water, intermediate water, and in the surf zone. Such models are able to simulate the wave breaking without the definition of any "a priori" criterion to locate the beginning of the breaking zone. Complex hydrodynamic phenomena are produced by the interaction between waves and coastal structures, in which the presence of the structure can lead to velocity fields with three-dimensional features that are locally important. The phenomenon of the undertow consists in a nearshore circulation current in which the velocities at the free-surface are onshore directed, while the near-bed velocities are offshore directed. This phenomenon is characterized by a full three-dimensionality. For a representation of the turbulent phenomena, in which the vortex structures have fully three-dimensional features, a complete representation of the hydrodynamic quantities, which involve vertical direction, is needed. The phenomena studied in this special issue are characterized by a high variability of hydrodynamic quantities along the vertical direction. Therefore, the aforementioned studies are carried out by fully solving three-dimensional motion equations.

There are two other elements that characterize the papers of this special issue: (1) a simulation's numerical procedures for the abovementioned motion equations, which are located in the volume of fluid (VOF) methodology and defined by shock-capturing schemes, and (2) the different formalizations of motion equations in a time-dependent generalized curvilinear coordinate system.

The models presented in [15-19] use more recent VOF methodology for their three-dimensional free surface flow simulations. In this technique, the vertical fluxes cross calculation cells. The free surface is tracked using the interpolated means of cell-averaged values in the water depth. By using this technique, it is possible to obtain excellent simulations of breaking waves. In [15], the authors develop a three-dimensional numerical model to study the application of the step with non-uniform heights at the bottom. [16] presents a numerical investigation concerning the impact emerged vegetation has on non-breaking, breaking, and broken wave propagation through vegetation over flat and sloping beds. The investigation uses the Reynolds-averaged Navier-Stokes (RANS) equations coupled with a VOF surface-capturing method. [17] proposes a three-dimensional numerical simulation of dam discharge and flood routing in the Wudu reservoir. In [18], the authors propose a three-dimensional simulation of wave transmission for low-crested and submerged breakwaters. [19] develops a three-dimensional numerical model for water wave simulations using the moving boundary method, which is based on RANS equations and the volume of fluid method. 
A numerical procedure (for the three-dimensional simulation of the wave motion) alternative to VOF methodology is defined in the context of shock-capturing schemes, in which the vertical coordinate varies in time to follow free surface movements. The irregular and time-varying physical domain is mapped into a fixed and regular computational one; it has a rectangular prismatic shape. As noted above, some of the most recent models based on this approach show a coordinate transformation in the vertical direction (named sigma coordinate transformation) by which the Cartesian vertical coordinate is expressed as a function of a moving vertical coordinate, $\sigma$, which adjusts to free surface motions. By doing so, the free surface is always located at the upper computational boundary. Furthermore, the pressure boundary condition at the free surface can be assigned to zero without any approximation. When motion equations are expressed in terms of conserved variables, the high-order shock captures numerical schemes that guarantee convergence to a correct weak solution and are then able to track the actual location of the breaking wave without requiring any criterion. A recent development for three-dimensional models is located in the $\sigma$ coordinate context, which adopts a shock-capturing scheme that can simulate breaking waves in the surf zone [20-27].

The second element that characterizes the papers in this special issue are related to two different formalizations of motion equations. In the first case, the motion equations are expressed in terms of Cartesian-based unknown variables on a time-dependent generalized curvilinear coordinate system; in the second case, the motion equations are expressed in a contravariant formulation and solved on a time-dependent generalized curvilinear coordinate system.

\section{Overview of the Special Issue}

Ref. [1] presents a turbulence model for wave breaking simulations. In the wave motion simulation near the shoreline, the initial wave breaking point and wave height reduction caused by a breaking wave must be simulated to correctly represent the undertow and sediment transport phenomena. Three-dimensional shock-capturing models presented in the literature show how the vertical coordinate is transformed in order to follow the free surface movements. These models can correctly represent the initial wave breaking point without any turbulence model and without any "a priori" criterion. However, these models underestimate the energy dissipation due to the wave breaking and consequently do not correctly simulate wave height in the surf zone. Thus, [1] presents a new model where turbulent kinetic energy and its dissipation rates $(\mathrm{k}-\varepsilon)$ are demonstrated. This model is located in the unsteady Reynolds averaged Navier-Stokes equations (URANS) and are a modification of standard $\mathrm{k}-\varepsilon$ models. The authors demonstrate that the proposed model is able to correctly dissipate the turbulent kinetic energy due to wave breaking. Therefore, it is able to correctly simulate the initial wave breaking point and the wave height in the surf zone.

Ref. [2] conducts a study on regular wave propagation over a sloping bed under the action of steady wind. The paper stems from the consideration that wind action produces fluctuations on the water's surface with continuous momentum and energy transfer in air-sea interaction. Many papers in the literature focus on wind wave generation rather than what effects wind has on wave motion already developed in coastal areas. The wind and wave fields are simulated by solving motion equations in a vertical domain using a VOF method and a large eddy simulation (LES) approach. This directly simulates the vortex structure, which contains most of the turbulent kinetic energy. The study leads to interesting conclusive considerations. The wind action induces earlier wave breaking and the initial wave breaking point migrates in an offshore direction. The wind effects on turbulence structure can be summarized in the enhancement of the coherence peak and turbulence intensity in the low-frequency range. In the high-frequency range, spectral density is larger without wind. The turbulent kinetic energy near the water's surface becomes significantly larger when wind increases. In the surf zone, an increase in wind velocity produces an undertow increase. A wind-induced setup adds to the wave setup and contributes to the undertow's acceleration for lost water carried shoreward after wave breaks and wind. 
In [3], the authors propose a study of waves overtopping coastal barriers. The overtopping of a coastal barrier by waves compromise the structural integrity of a barrier. Furthermore, the wave overtopping causes the presence of wave motion in the leeside of the barriers, i.e., in the zone that the structure is intended to protect. The phenomenon of wave overtopping has a complex nature and different aspects have to be taken into account to give it a proper representation. Some of the complex aspects of wave overtopping include wave transformation from deep to shallow water; three-dimensionality of the velocity fields; wave breaking; and wave runup and run-down over the coastal structure. In the model proposed by [3], three-dimensional motion equations are expressed in Cartesian-based unknown variables via a time-dependent generalized curvilinear coordinate system. Thus, wave transformation and three-dimensional velocity fields can be caught. Wave breaking is simulated using a shock-capturing scheme. Moreover, the authors propose a technique to simulate wet-dry interface propagation to represent wave fronts propagating over the structure. They conduct an experimental study to validate the numerical model. More specifically, they implement a nonintrusive and continuous-in-space image-analysis technique, which allowed them to catch the wave front advancing over the structure and measure shallow water wave fronts.

Ref. [4] detail a three-dimensional numerical simulation form for a tsunami wave propagating in a reservoir as a result of landslide that makes a solid mass detach from the mountain side and slide into the reservoir. The well-known case of the Vajont dam, in which nearly 2000 people lost their lives in 1963 following the overtopping of the Vajont dam after a solid mass fell from the reservoir slopes, demonstrated that such events, despite being infrequent, can be catastrophic. The considerable dimensions of artificial reservoirs and solid-liquid masses that move during these phenomena limit experimental studies on landslide tsunamis to laboratory scale model simulations. By contrast, a numerical approach allows for full-scale simulations. [4] represents an example of a three-dimensional numerical model for free-surface flow to a full-scale artificial reservoir. The numerical simulations are carried out using a model that solves the RANS Equations and adopts a $\mathrm{k}-\varepsilon$ turbulence model. The update of the free surface is carried out using a VOF method. The adopted method is validated by numerically reproducing laboratory tests of subaerial and submarine landslide-generated tsunamis. Once validated, the three-dimensional proposed model is applied to a real test-case simulation with engineering interest, which regards the simulation of a tsunami inside a Chinese artificial reservoir in which one of the slopes has a high landslide risk. The numerical simulations show that, given the proximity between a dam and a high-risk zone, the landslide-generated tsunami wave may exceed the dam crest and thus overtopping can occur. The authors show that with a proper grid refinement strategy (more refined in the zone in which the tsunami wave is generated and less refined in the wave propagation zone), the model provides accurate results.

Ref. [5] presents an application case concerning the Cetraro harbor in Italy. The problem of the Cetraro harbor is characterized by the fact that obliquely incident wave trains cause longshore currents that produce siltation at the harbor's entrance. By using three-dimensional simulations, the authors demonstrate that in the coastal area in front of the Cetraro harbor entrance, the abovementioned longshore current is modified by the complex shape of the shoreline and consequently produces an anticlockwise vortex structure. The authors show that the solid particles, which in the surf zone are picked-up by wave-induced turbulence, can be carried out by the longshore current and vortex structure up to the area in front of the Cetraro harbor entrance. In this area, where the wave energy is significantly reduced, solid particles tend to settle and produce siltation by the harbor entrance. The effects produced on the currents and vortex structure by a new designed breakwater are verified via three-dimensional hydrodynamic simulations. [5] demonstrates a new designed breakwater influence for wave field propagation, producing a modification in the hydrodynamic field by erasing vortex structure.

Some considerations on turbulence models-many of which are adopted in the special issue's papers-must be done. In a simulation for wave motion near shoreline, the initial wave breaking point and the reduction of wave height after wave breaking must be adequately represented. Wave breaking can be simulated using a three-dimensional motion equation, which is solved via a shock-capturing 
model. Shock-capturing models are located in the $\sigma$-coordinate context and use a simple Smagorinsky turbulence model to reduce numerical spurious oscillations. Moreover, these models are able to correctly represent the location of the initial wave breaking point. However, these models entrust to the numerical scheme a task to reduce wave height in the surf zone and consequently are unable to adequately represent the transfer of energy to turbulence scales. Thus, it is necessary to introduce appropriate turbulence models in motion equations that represent the actual energy dissipation due to wave breaking, as well as the actual reduction of wave height in the surf zone. In general, the vortex structures associated to the stochastic fluctuations in the surf zone are superimposed on unsteady quasi-periodic hydrodynamic structures. Standard $\mathrm{k}-\varepsilon$ turbulence models located in RANS equations expel from velocity fields unsteady quasi-periodic hydrodynamic structures, as well as vortex structures associated with stochastic fluctuations. These standard models produce excessive energy dissipation after an initial wave breaking point by overestimating the turbulent stress tensor. Thus, they consequently produce non-physical wave height reduction in the surf zone. Further, [1] proposes a turbulence model located in URANS equations. The abovementioned model consists of a modification for the standard $\mathrm{k}-\varepsilon$ model. The closure relation for turbulence stress tensor considers effects produced by the stochastic vortex structures and allows for the direct simulation of unsteady quasi-periodic hydrodynamic structures. The additional turbulence model, presented in [4], describes the simulation for a landslide-generated tsunami. The model uses the closure relation or a turbulence stress tensor and a $\mathrm{k}-\varepsilon$ model, which follows the re-normalization group turbulent model (RNG).

In [2], the authors simulate wind-produced effects on wave propagation and consider what the effects wind has on the turbulence. The authors use an LES approach in which the motion equations are explicitly filtered over space by a convolution integral. In the study of wave motion, numerical simulations carried out with an LES approach are rarely used since they have higher computational costs than those in RANS equations. Although LES does resolve all spatial and temporal turbulence scales, the spatial scales that contain most of the turbulent kinetic energy are directly simulated. Thus, the turbulence model is used to represent only the effect produced on the resolved scale of motion using the smallest spatial and temporal scales associated with turbulent structures smaller than calculation cells. In order to avoid too much kinetic energy dissipation from the resolved scale of motion, LES turbulence models (based on the Smagorinsky model) use the LES as a function of twice the dimension of the grid cell, which represents the spatial filter width. Since the spatial discretization of the physical domain acts like an implicit filter that can remove the smallest scale of motion from the numerical solution, the LES requires spatial and time discretization steps small enough to directly simulate the turbulent structures that contain most of the turbulent kinetic energy. Near the bottom of the free surface, the dimensions of such turbulent structures are small enough to require significant refinement of the calculation grid, both in vertical and horizontal directions. [4] represents an example of LES used for wave motion, in which this approach evaluates the effect of wind on the turbulence produced by wave breaking.

A few considerations are made on numerical procedures adopted in [1-5]. In [2,4], the authors simulate a three-dimensional free surface flow by using a VOF technique. VOF consists of a two-phase flow numerical simulation (air and water). In two-phase flow equations, the unknown variables are volume fractions and velocities of aeriform and liquid phases. The volume fraction and velocity are determined in each calculation cell by resolving motion equations for each phase. In cells crossed by the free surface, the volume fractions of both phases do not equal zero. In VOF models, the free surface is tracked by interpolated means of cell-averaged values to water depth. By using this technique, the authors obtain excellent simulations for wave breaking.

A second category of models used for free surface flow three-dimensional simulations maps a physical domain that varies in time along the vertical direction to follow free surface movements ( $\sigma$-coordinate transformation) to a computational domain where calculation cells have a rectangular prismatic shape. A generalized $\sigma$-coordinate model uses motion equations to express Cartesian-based unknown variables on a time-dependent boundary conforming generalized curvilinear coordinate 
systems where only the vertical coordinate follows the free surface evolution [1,3]. Motion equations are solved by using a shock-capturing numerical scheme that correctly simulates the initial breaking point without introducing any "a priori" criterion and reduces wave height in the surf-zone. In this model, the kinematic and pressure boundary conditions on the upper face of the free surface calculation cell are correctly assigned.

A further generalization of the three-dimensional shock-capturing model consists of expressing motion equations in a contravariant formula on a time-dependent, generalized curvilinear coordinates system, in order to study flows in complex geometries [5].

\section{Conclusions}

This special issue concerns the simulation of wave fields and wave-induced velocity fields in coastal areas. The five original papers present theoretical aspects of the models, specific aspects of the adopted numerical schemes, and numerical model applications to practical engineering case studies. In every papers, emphasis is placed on the need to adopt three-dimensional simulations for free surface flows. Three different approaches are followed for the aforementioned three-dimensional simulations. In the first approach, the VOF technique is used. In the second, three-dimensional motion equations are expressed in Cartesian-based unknown variables in a time-dependent, generalized coordinate system. In the third, three-dimensional motion equations are expressed in contravariant formulations in a time-dependent, generalized curvilinear coordinate system, which can reproduce complex coastal regions. Turbulence modelling is defined by a turbulent stress tensor, which is expressed as a function of turbulent kinetic energy and dissipation rate of turbulent kinetic energy, $\mathrm{k}-\varepsilon$, modified with respect to the $\mathrm{k}-\varepsilon$ standard formulation to correctly represent the total dissipation rate of turbulent kinetic energy for wave breaking. Further, it is defined by the LES to simulate the effects of wind on the propagation of waves over a sloping bed and on wave breaking.

Funding: This research received no external funding.

Conflicts of Interest: The author declares no conflict of interest.

\section{References}

1. Cannata, G.; Palleschi, F.; Iele, B.; Gallerano, F. A modified k $-\varepsilon$ turbulence model for a wave breaking simulation. Water 2019, 11, 2282. [CrossRef]

2. Jiang, C.; Yang, Y.; Deng, B. Study on the nearshore evolution of regular waves under steady wind. Water 2020, 12, 686. [CrossRef]

3. Cannata, G.; Tamburrino, M.; Ferrari, S.; Badas, M.G.; Querzoli, G. Numerical and experimental investigation of wave overtopping of barriers. Water 2020, 12, 451. [CrossRef]

4. Li, G.; Chen, G.; Li, P.; Jing, H. Efficient and accurate 3-D numerical modelling of landslide tsunami. Water 2019, 11, 2033. [CrossRef]

5. Cannata, G.; Palleschi, F.; Iele, B.; Cioffi, F. A three-dimensional numerical study of wave induced currents in the Cetraro harbor coastal area (Italy). Water 2020, 12, 935. [CrossRef]

6. Shi, F.; Kirby, J.T.; Harris, J.C.; Geiman, J.D.; Grilli, S.T. A high-order adaptive time-stepping TVD solver for Boussinesq modelling of breaking waves and coastal inundation. Ocean Model. 2012, 43, 36-51. [CrossRef]

7. Tonelli, M.; Petti, M. Shock-capturing Boussinesq model for irregular wave propagation. Coast. Eng. 2012, 61, 8-19. [CrossRef]

8. Roeber, V.; Cheung, K.F. Boussinesq-type model for energetic breaking waves in fringing reef environments. Coast. Eng. 2012, 70, 1-20. [CrossRef]

9. Chen, Q.; Kirby, J.T.; Dalrympe, R.A.; Shi, F.; Thornton, E.B. Boussinesq modeling of longshore currents. J. Geophys. Res. 2003, 108, 18-26. [CrossRef]

10. Liu, W.; Ning, Y.; Zhang, Y.; Zhang, J. Shock-capturing Boussinesq modelling of broken wave characteristics near a vertical seawall. Water 2018, 10, 1876. [CrossRef]

11. Zhang, S.; Zhu, L.; Li, J. Numerical simulation of wave propagation breaking, and setup on steep fringing reefs. Water 2018, 10, 1147. [CrossRef] 
12. Pan, J.; Shen, H.T. Tsunami intrusion and river ice movement. Water 2019, 11, 1290. [CrossRef]

13. Liu, W.; Shao, K.; Ning, Y. A study of the maximum momentum flux in the solitary wave run-up over back-reef slopes based on a Boussinesq model. J. Mar. Sci. Eng. 2019, 7, 109. [CrossRef]

14. Lo Re, C.; Manno, G.; Ciraolo, G. Tsunami propagation and flooding in Sicilian Coastal Areas by means of a weakly dispersive Boussinesq model. Water 2020, 12, 1148. [CrossRef]

15. Li, D.; Yang, Q.; Ma, X.; Dai, G. Case study on application of the step with non-uniform heights at the bottom using a numerical and experimental model. Water 2018, 10, 1762. [CrossRef]

16. Zou, X.; Zhu, L.; Zhao, J. Numerical simulations of non-breaking, breaking and broken wave interaction with emerged vegetation using Navier-Stokes equations. Water 2019, 11, 2561. [CrossRef]

17. Rong, Y.; Zhang, T.; Peng, L.; Feng, P. Three-dimensional numerical simulation of dam discharge and flood routing in Wudu reservoir. Water 2019, 11, 2157. [CrossRef]

18. Li, X.; Zhang, W. 3D numerical simulation of wave transmission for low-crested and submerged breakwaters. Coast. Eng. 2019, 152, 103517-103531. [CrossRef]

19. Jia, W.; Liu, S.; Li, J.; Fan, Y. A three-dimensional numerical model with an L-type wave-maker system for water wave simulations by the moving boundary method. Water 2020, 12, 161. [CrossRef]

20. Ma, G.; Shi, F.; Kirby, J.T. Shock-capturing non-hydrostatic model for fully dispersive surface wave processes. Ocean Model. 2012, 43, 22-35. [CrossRef]

21. Young, C.C.; Wu, C.H. A $\sigma$-coordinate non-hydrostatic model with embedded Boussinesq-type-like equations for modelling deep-water waves. Int. J. Numer. Methods Fluids 2010, 63, 1448-1470.

22. Bradford, S.F. Non-hydrostatic model for surf zone simulation. J. Waterw. Port. Coast. 2011, 137, $163-174$. [CrossRef]

23. Derakhti, M.; Kirby, J.T.; Shi, F.; Ma, G. NHWAVE: Consistent boundary conditions and turbulence modeling. Ocean Model. 2016, 106, 121-130. [CrossRef]

24. Derakhti, M.; Kirby, J.T.; Shi, F.; Ma, G. Wave breaking in the surf zone and deep-water in a non-hydrostatic RANS model. Part 1: Organized wave motions. Ocean Model. 2016, 107, 125-138. [CrossRef]

25. Derakhti, M.; Kirby, J.T.; Shi, F.; Ma, G. Wave breaking in the surf zone and deep-water in a non-hydrostatic RANS model. Part 2: Turbulence and mean circulation. Ocean Model. 2016, 107, 139-150. [CrossRef]

26. Cannata, G.; Gallerano, F.; Palleschi, F.; Petrelli, C.; Barsi, L. Three-dimensional numerical simulation of the velocity fields induced by submerged breakwaters. Int. J. Mec. 2019, 13, 1-14.

27. Hsu, C.J.; Hung, C. Three-dimensional wave-induced dynamic response in anisotropic poroelastic seabed. Water 2020, 12, 1465. [CrossRef] 